\title{
Capacity-Achieving Techniques in Nonlinear Channels
}

\author{
Sergei K. Turitsyn \\ Aston Institute of Photonic Technologies, Aston University, B4 7ET, Birmingham, UK, \\ email: s.k.turitsyn@aston.ac.uk
}

\begin{abstract}
Many of the current optical transmission techniques were developed for linear communication channels and are constrained by the fibre nonlinearity. This paper discusses the potential for radically different approaches to signal transmission and processing based on using inherently nonlinear techniques.
\end{abstract}

\section{Introduction}

The modern optical fibre transmission systems and technologies that are the largest contributors to global data traffic are facing challenges due to the nonlinear properties of fibre channels [1-7]. It is important to recognize that most of these concepts and techniques have been developed for linear communication channels, such as e.g., radio channels. While this provides access to a vast number of already developed technologies, these methods may be not optimal for nonlinear communication channels, limiting achievable transmission rates and spectral efficiency. The impact of nonlinearity on capacity of fibre channel has been a subject of intensive studies in recent years [1-15].

Although nonlinearity is an essential component in the design of advanced fibre communication systems, it is often shunned by engineers because of its intractability. However, mastering the nonlinear effects can translate into a significant increase in the capacity of communications systems.

This paper will outline several new approaches that aim to develop a practical framework for coding, modulation and transmission techniques based on a mathematical theory of integrable nonlinear systems [16-25], and also aim to design nonlinear channels with constructive nonlinearity $[26,27]$.

\section{Nonlinear Fourier Transform}

The lossless nonlinear Schrödinger equation (NLSE) (written here in dimensionless form) is a principal model of the nonlinear fibre channel [5] (term $\eta$ accounts for an effective distributed noise; we have omitted noise analysis below):

$$
i \frac{\partial q}{\partial z}+\frac{1}{2} \frac{\partial^{2} q}{\partial t^{2}}+|q|^{2} q=\eta(z, t)
$$

This lossless NLSE model can be derived under certain conditions by averaging over periodic gain and loss variation [20]. Moreover, recent demonstrations of a quasi-lossless fibre span $[28,29]$ has shown that gain/loss variations can be compensated continuously along the fibre. Eq. (1) belongs to the class of the so-called integrable nonlinear systems [16-20].

In simple terms, the integrability of NLSE means that the nonlinear field evolution described by Eq. (1) can be presented in a special basis (that is nonlinear analogue of the Fourier transform $(\mathrm{FT})$ ), within which the dynamics of individual "orthogonal nonlinear modes"' is effectively linear without any mode interactions. A powerful method of the inverse scattering transform - the nonlinear Fourier transform (NFT) method [16 20] can be applied to find the solutions for Eq. (1). A standard FT approach to linear equation converts the initial field given in time into the frequency domain (forward FT): $A(t, z=0) \Rightarrow \tilde{A}(\omega, z=0)$, the spectral domain components are non-interaction (orthogonal) and evolution changes can be easily found for each component: $\tilde{A}(\omega, 0) \Rightarrow \tilde{A}(\omega, L)$. After that, backward FT gives the field evolution in time domain: $\tilde{A}(\omega, L) \Rightarrow A(t, L)$.

Within the NFT method as applied to Eq. (1), the first step (decomposition of initial signal field $q(t, z=0) \Rightarrow \mathrm{Z}(\lambda, z=0)$ into spectral data, forward NFT) is to solve a linear spectral Zakharov-Shabat problem (ZSP) [16-20]:

$$
\begin{aligned}
& \frac{d f}{d t}=-i \lambda g+q(t) f, \\
& \frac{d g}{d t}=-q^{*}(t) f+i \lambda g,
\end{aligned}
$$

Here, $\lambda=\xi+i \sigma$ is a (generally complex) eigenvalue, and the function $q(t)$ is the input signal. The forward NFT operation corresponds to mapping of the initial field, $q(t)=q(t, z=0)$, onto a set of scattering data: $\Sigma=\left\{r(\xi)=\lim _{t \rightarrow \infty}\left[e^{-2 i \lambda t} \frac{g(\lambda, t)}{f(\lambda, t)}\right], \xi \in R ;\left\{\lambda_{n}, C_{n}\right\}\right\}$ (see [16-25] for details; below, we have omitted discrete scattering data $\left\{\lambda_{n}, C_{n}\right\}$ corresponding 
to solitons). The nonlinear spectrum $r(\xi)$ is the nonlinear analogue of the Fourier spectrum, tending (after some rescaling) to the standard FT of $\mathrm{q}(\mathrm{t})$ in the low power limit. The evolution of $r(\xi)$ is trivial: $r(\xi, L)=r(\xi, L) e^{2 i \xi^{2} L}$. Therefore, the orthogonality of nonlinear normal modes is preserved during the signal propagation.

The backward NFT maps the scattering data for example at $z=L, \Sigma(\lambda, z=L)$ - onto the field $q(t, L)$. This is achieved via the GelfandLevitan-Marchenko equation (GLME) for the unknown function $\mathrm{K}\left(\mathrm{t}, \mathrm{t}^{\prime}\right)$ [16-20]:

$$
\begin{aligned}
& K\left(t, t^{\prime}\right)+F\left(t+t^{\prime}\right)+ \\
& \iint_{-\infty}^{t} K(t, y) F^{*}(y+x) F\left(x+t^{\prime}\right) d y d x=0
\end{aligned}
$$

Here, $F(t)=\frac{1}{2 \pi} \int d \xi r(\xi, L) e^{-i \xi t}$ is the linear FT of $r(\xi)$. The solution of GLME for $K\left(t, t^{\prime}\right)$ defines the backward NFT that recovers $q(t, L)=2 K(t, t)$.

Note that the fibre nonlinear effects, such as self-phase modulation, cross-phase modulation and four-wave-mixing, are included in the NFT [16-24]. This means that, in a proper nonlinear basis, there is no any nonlinear cross-talk [1624] and the linear channel capacity can be potentially approached.

This property constitutes the general idea of the eigenvalue communication first introduced in [21], the essence of which is to use invariant ZSP eigenvalues (orthogonal nonlinear modes of NLSE) to encode and transmit information. The application of NFT-decomposition opens fundamentally new possibilities for advanced coding and modulation, which are resistant to nonlinear transmission impairments.

Note that, in [21], only the discrete part of the ZSP spectrum was considered. This discrete spectrum corresponds to the soliton part of the NLSE solution. In [23-25], the idea of NFT was studied in a context of non-soliton eigenvalue communications. The NFT digital signal processing is based on the encoding of information directly onto the continuous nonlinear signal spectrum that evolves linearly along the transmission in a nonlinear integrable channel-the nonlinear eigenvalue division multiplexing [25]. By applying the NFT technique, it is possible to develop a new signal processing routine for compensating nonlinear distortions [21-25]. The main challenge is to develop fast numerical algorithms for solving ZSP and GLME - analogue of fast FT [30,31].

\section{Nonlinear channels with noise squeezing}

Another interesting possibility of a positive use of nonlinearity is to insert in-line (after some amplifiers) nonlinear elements with regenerative functions. This creates new nonlinear channels. The high capacity of such nonlinear channels can be achieved when noise is suppressed (squeezed) using nonlinear elements; that is, the regenerative function not available in linear systems [26,27].

The regenerative mapping is schematically illustrated by Fig. 1, in which four constellation points are mapped to the effective nonlinear potential (formed by the in-line nonlinear elements) that prevent noise from growing unrestricted (as in the corresponding linear channel). An important new feature introduced by the nonlinear mapping is the possibility of continuous nonlinear filtering of noise without requiring a hard decision.

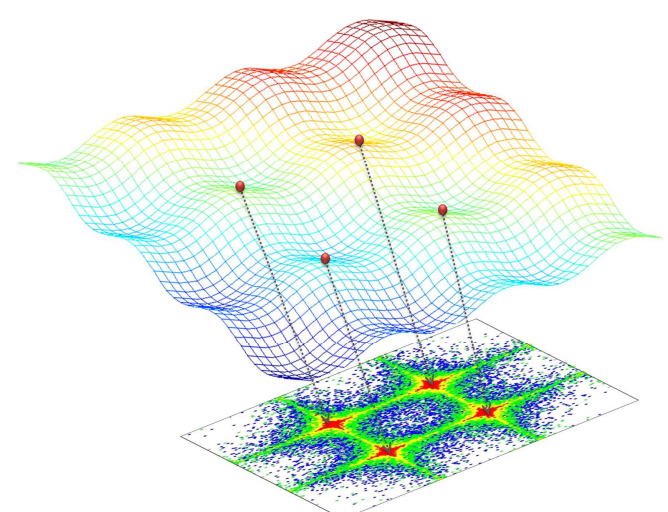

Fig.1: Schematic illustration of effective noise squeezing nonlinear potential created by nonlinear elements. Rectangular $M=4$ constellations after $R$ consequent nonlinear tmaps interleaved with noise are shown (below) at the output of the $R$-th nonlinear filter (blue $-\mathrm{R}=1$, green $R=5$, yellow $R=10$ and red $R=20$ );for details, see [27].

We should stress the difference between the considered nonlinear in-line processing and full regeneration involving receiver and retransmitter pairs at each regeneration node. Whenever the nonlinear transformation has multiple fixed points, the consequent interleaving of the accumulating noise with the nonlinear filter produces effective suppression of the noise. The effective washboard potential that is created quantizes the signal and improves transmission, with a consequent increase in capacity. Details can be found in [26,27].

\section{Conclusions}

From a practical standpoint, the fibre nonlinearity greatly increases the difficulty of understanding system behavior. On the other hand, new techniques may be developed that cannot be realized in linear systems. Moreover, 
use of constructive nonlinearity with regenerative (for example, noise squeezing) functions can create communication channels that are fundamentally different from the linear AWGN channel.

The introduced class of regenerative mapping channels has a high information capacity that is achieved by noise squeezing due to the introduced nonlinear filters that create attraction regions around the stable alphabet.

\section{Acknowledgements}

I would like to acknowledge the coauthors in the publications overviewed in this paper and collaborators: K. S. Turitsyn, Y. Prilepskiy, M. Sorokina, S. Derevyanko, E. G. Turitsyna, S. K.J. Blow, I. Gabitov, S. T. Le, L. Frumin, and E. Podivilov, as well as the support from the EPSRC project UNLOC EP/J017582/1.

\section{References}

[1] A. Splett, C. Kurtzke, and K. Petermann, "Ultimate transmission capacity of amplified optical fiber communication systems taking into account fiber nonlinearities," Tech. Digest of ECOC, MoC2.4 (1993).

[1] P. P. Mitra and J. B. Stark, "Nonlinear limits to the information capacity of optical fibre communications," Nature, 411, 1027 (2001).

[2] E. B. Desurvire, "Capacity demand and technology challenges for lightwave systems in the next two decades," J. Lightwave Technol., 24, 4697 (2006).

[3] R.-J. Essiambre, G. Foschini, G. Kramer, and P. Winzer, "Capacity limits of information transport in fiber-optic networks," Phys. Rev. Lett.101, 163901 (2008).

[4] A. D. Ellis, J. Zhao, and D. Cotter, "Approaching the non-linear Shannon limit," J. Lightwave Technol.28, 423 (2010).

[5] R.-J. Essiambre, G. Kramer, P. J. Winzer, G. J. Foschini, B. Goebel, "Capacity limits of optical fibernetworks," J. Lightw. Technol. 28, 662 (2010).

[6] D. J. Richardson, "Filling the light pipe," Science, 330, 327 (2010).

[7] R. Tkach, "Scaling optical communications for the next decade and beyond," Bell Labs Tech. J.14(4), 3 (2010).

[8] K. S. Turitsyn et al.,"Information capacity of optical fiber channels with zero average dispersion," Phys. Rev. Lett. 91, 203901-1 (2003).

[9] R. I. Killey and C. Behrens, "Shannon's theory in nonlinear systems," J. Mod. Opt., 58, 1 (2011).

[10]A. Mecozzi and R.-J. Essiambre, "Nonlinear Shannon limit in pseudo-linear coherent systems," J. Lightwave Technol.30, 2011 (2012).

[11] "Impact of nonlinearities on fiber-optic communication systems", Ed. By S. Kumar, (Springer, 2011).

[12]E. Agrell, "The channel capacity increases with power", preprint arXiv:1108.0391v2 (2012).

[13] E Agrell, M Karlsson, "DM channel capacity and its dependence on multichannel adaptation models", Proc. OFC, OTu3B.4 (2013)
[14]E Agrell, "Nonlinear fiber capacity", Proc. ECOC, London (2013)

[15]E Agrell, A Alvarado, G Durisi, M Karlsson, "Capacity of a Nonlinear Optical Channel with Finite Memory" arXiv preprint arXiv:1403.3339, 2014

[16]V. E. Zakharov and A. B. Shabat, "Exact theory of twodimensional self-focusing and one-dimensional selfmodulationof waves in nonlinear media," Soviet PhysicsJETP 34, 62-69 (1972).

[17]S.V. Manakov," "On the theory of two-dimensional stationary self focussing of electromagnetic waves",Sov. Phys. JETP 38(2), 248-253 (1974).

[18]M. J. Ablowitz, D. Kaup, A. Newell, and H. Segur, "The inverse scattering transform Fourier analysis for nonlinear problems," Stud. Appl. Math. 53, 249 (1974).

[19] V. E. Zakharov, S. V. Manakov, S. P. Novikov, and L. P. Pitaevskii, Theory of Solitons. The Inverse Scattering Method (Consultants Bureau, 1984).

[20]A. Hasegawa and Y. Kodama, Solitons in Optical Communications (Oxford University Press, 1995).

[21]A. Hasegawa and T. Nyu, "Eigenvalue communication," J. Lightwave Technol. 11, 395-399 (1993).

[22]M. I. Yousefi and F. R. Kschischang, "Information transmission using the nonlinear Fourier transform, Part I: Mathematical tools," http://arxiv.org/abs/1202.3653, "Part II: Numerical methods," Apr. 2012, online: http://arxiv.org/abs/1204.0830; "Part III: Spectrum modulation," Feb. 2013, http://arxiv.org/abs/1302.2875, accepted IEEE Trans. Inf. Theory.

[23]E. G. Turitsyna and S. K. Turitsyn, Digital signal processing based on inverse scattering transform, Opt. Lett. 38, 4186 (2013).

[24] J. E. Prilepsky, S. A. Derevyanko, and S. K. Turitsyn,Nonlinear spectral management: Linearization of the lossless fiber channel, Opt. Express21, 24344 (2013).

[25]J. E. Prilepsky, S. A. Derevyanko, K.J. Blow, I. Gabitov, and S. K. Turitsyn, "Nonlinear inverse synthesis and eigenvalue division multiplexing in optical fiber channels", Phys. Rev. Lett., accepted (2014)

[26] K. S. Turitsyn and S. K. Turitsyn, "Nonlinear communication channels with capacity above the linear Shannon limit," Opt. Lett.37, 3600 (2012).

[27]M. A. Sorokina and S. K. Turitsyn, "Regeneration limit of classical Shannon capacity", Nature Communications 5, 3861 (2014).

[28]J D Ania-Castanonet al.,"Ultralong Raman fiber lasers as virtually lossless optical media”, Phys. Rev. Lett. 96, 023902 (2006).

[29]T J Ellingham et al., "Quasi-Lossless Optical Links for Broad-Band Transmission and Data Processing", IEEE Photon. Technol. Lett. 18(1), 268 (2006).

[30] S. Burtsev, R. Camassa, and I. Timofeyev, J. Comput. Phys. 147, 166 (1998).

[31] S.Wahls and H.V.Poor, "Fast Numerical Nonlinear Fourier Transforms", in Proc. of ICASSP IEEE, 5780 (2013); http://dx.doi.org/10.1109/ICASSP.2013.6638772; submitted to IEEE Trans. Inf. Theory (2013). 